\title{
A guide to multiple-sample structural equation modeling
}

\author{
RICHARD G. LOMAX \\ Louisiana State University, Baton Rouge, Louisiana
}

\begin{abstract}
The application of structural equation modeling to the investigation of social phenomena has increased in recent years. Whereas description and application of the LISREL methodology to the single-sample situation abound, such is not the case for the multiple-sample situation (i.e., simultaneous analysis of independent samples). The multiple-sample case has many possible applications in psychology (e.g., the analysis of experimental, nonexperimental, cross-sectional, and longitudinal data). The guide features descriptions of (1) the simple multiple-sample case, in which equality constraints may be imposed on the covariance structure of the measurement and/or structural equation models across samples, (2) the structured-means multiplesample case, in which constraints may be additionally imposed on the mean structure of these models across samples, and thereby allow an assessment of group differences, and (3) a sequential strategy for dealing with the multiple-group situation, illustrated through a model of public and private schooling.
\end{abstract}

The application of structural equation modeling to the investigation of social phenomena has increased in recent years (e.g., Bentler, 1980). The LISREL (linear structural relationship) model (Jöreskog, 1978) consists of the measurement and structural equation models. The measurement model describes the measurement of unobservable latent variables by observable indicators or manifest variables and allows for evaluation of the measurement properties of the indicators. In the structural equation model, a set of general linear equations describes the theoretical causal relationships among the latent variables. The LISREL methodology frequently has been described and applied in the single-sample situation, but not in the multiple-sample situation (e.g., simultaneous analysis of independent samples). The multiple-sample case has many possible applications in psychology (e.g., the analysis of experimental, nonexperimental, cross-sectional, and longitudinal data). The purpose of this paper is to (1) describe in detail the theory supporting the multiple-sample LISREL model, and (2) construct a guide to model fitting for prospective LISREL users. Refer to Lomax (1982) for a similar discussion of the single-sample case, which is not dealt with here.

\section{THEORY OF THE MULTIPLE-SAMPLE LISREL MODEL}

This section includes a discussion of (1) the simple multiple-sample case, in which equality constraints are imposed on the covariance structure of the measurement

The author's mailing address is: College of Education, Louisiana State University, Baton Rouge, Louisiana 70803. and/or structural equation models across samples, and (2) the structured-means multiple-sample case, in which constraints are additionally imposed on the mean structure of the models across samples, and thereby allow an assessment of sample or group differences.

\section{Simple Multiple-Sample Model}

In the structural equation model, let $\eta(\mathrm{mx} 1)$ and $\xi(n \times 1)$ be random vectors of the latent dependent and independent variables, respectively, so that a system of linear structural equations is

$$
\eta=\mathrm{B}(\mathrm{g}) \eta+\Gamma(\mathrm{g}) \xi+\zeta,
$$

where $B(m \times m)$ and $\Gamma(m \times n)$ are matrices of structure coefficients, $\zeta(m \times 1)$ is a random vector of residuals due to equation errors, and $g=1, \ldots, G$ identifies the sample of interest. The samples are assumed to be independently drawn from their respective poplations. Assume that $\mathrm{E}(\eta)=\mathrm{E}(\zeta)=\mathrm{E}(\xi)=0$, that $\zeta$ is uncorrelated with $\xi$, and that $I-B$ is nonsingular.

In the measurement model, since $\eta$ and $\xi$ are unobservable, let $y(p \times 1)$ and $x(q \times 1)$ be vectors of the observable indicator variables, so that

$$
\mathrm{y}=\Lambda_{\mathrm{y}}(\mathrm{g}) \eta+\epsilon
$$

and

$$
\mathrm{x}=\Lambda_{\mathrm{x}}(\mathrm{g}) \xi+\delta,
$$

where $\epsilon(\mathrm{p} \times 1)$ and $\delta(\mathrm{q} \times 1)$ are vectors of the measurement errors (uniquenesses) in $y$ and $x$, respectively. The vectors $y$ and $x$ are measured as deviations from their respective means. Let $\Lambda_{y}(\mathrm{~g})(\mathrm{p} \times \mathrm{m})$ and $\Lambda_{\mathrm{x}}(\mathrm{g})$ 
(q $x n$ ) be regression matrices of $y$ on $\eta$ and of $x$ on $\xi$, respectively. Assume that the errors of measurement $\epsilon$ are uncorrelated with the errors of measurement $\delta$, and that $\epsilon$ and $\delta$ are uncorrelated with $\eta$, $\xi$, and $\zeta$.

When the structural equation and measurement models are combined, let $\Phi(\mathrm{g})(\mathrm{n} \times \mathrm{n})$ and $\Psi(\mathrm{g})(\mathrm{m} \times \mathrm{m})$ be the variance-covariance matrices of $\xi$ and $\zeta$, respectively. Also, let $\Theta_{\epsilon}(\mathrm{g})(\mathrm{p} \times \mathrm{p})$ and $\Theta_{\delta}(\mathrm{g})(\mathrm{q} \times \mathrm{q})$ be the variance-covariance matrices of $\epsilon$ and $\delta$, respectively. A population variance-covariance matrix $\Sigma$ is constructed from the eight matrices, $\Lambda_{y}(\mathrm{~g}), \Lambda_{\mathrm{x}}(\mathrm{g}), \mathrm{B}(\mathrm{g}), \Gamma^{(\mathrm{g})}$, $\Phi(\mathrm{g}), \Psi(\mathrm{g}), \Theta_{\epsilon}(\mathrm{g})$, and $\Theta_{\delta}(\mathrm{g})$ (see Equation 4 in Lomax, 1982, for more detail). Elements of these eight matrices may consist of fixed, free, and constrained parameters, as in the single-sample case. However, if there are no constraints between the samples, then each sample may be analyzed separately. If there are constraints between the samples, then the analysis must be conducted on all samples simultaneously.

Identification and estimation of the parameters are essentially the same as in the single-sample case, although obviously at a more complex level. For model testing, a single chi-square value is reported regardless of the number of samples analyzed (i.e., an overall chi-square test). The number of degrees of freedom is equal to $1 / 2 G(p+q)(p+q+1)-s$, where $s$ is the total number of independent parameters for all samples. For each sample, three other goodness-of-fit indexes are reported in the LISREL program. The goodness-of-fit index (GFI) is a measure of the relative amount of the sample variancecovariance matrix $\mathbf{S}$ accounted for by the model. In contrast to the chi-square test, the GFI is independent of sample size and is rather robust with respect to violation of the normality assumption. The adjusted GFI (AGFI) adjusts for the number of degrees of freedom in the model. Both of these indexes are theoretically scaled between 0 and 1 . The root-mean-square residual is a measure of the average residual variances and covariances. These three sample-specific indexes are useful for assessing the goodness of fit of a given sample across various models.

If a particular model does not fit the data reasonably well, one may be interested in determining the inadequacies of the model. Two procedures are available for diagnosing a lack of fit. First, normalized residuals are reported for each element of the sample variancecovariance matrix. These may be thought of as $\mathrm{z}$ scores, such that residuals larger than, say, 2 should be examined for possible misspecification of the model. Second, modification indexes are reported for each nonfree parameter and are based on the derivatives of the fitting function (see Lomax, 1982). A modification index for a particular nonfree parameter indicates that if this parameter were allowed to become free, the chisquare value would decrease by the value of the index. Thus, large modification indexes would suggest ways in which the LISREL model might be relaxed by allowing the corresponding parameters to become free, and thus arrive at a better fitting model.

The LISREL system of structural equation modeling presently is implemented in the LISREL VI (Jöreskog \& Sörbom, 1983) computer program. Possible constrained models to test in the simple multiple-sample situation are described later.

\section{Structured-Means Multiple-Sample Model}

In the LISREL model defined by Equations 1,2, and 3 , all random variables are assumed to have 0 means. Here, the assumption is relaxed, such that a structure may be imposed on the mean parameters. The LISREL model is rewritten as

$$
\begin{aligned}
& \eta=\alpha(\mathrm{g})+\mathrm{B}(\mathrm{g}) \eta+\Gamma(\mathrm{g}) \xi+\zeta, \\
& \mathrm{y}=\nu_{\mathrm{y}}(\mathrm{g})+\Lambda_{\mathrm{y}}(\mathrm{g}) \eta+\epsilon, \\
& \mathrm{x}=\nu_{\mathrm{x}}(\mathrm{g})+\Lambda_{\mathrm{x}}(\mathrm{g}) \xi+\delta,
\end{aligned}
$$

where $\alpha, \nu_{\mathrm{y}}$ and $\nu_{\mathrm{x}}$ are vectors of constant intercept terms that no longer need be constrained to 0 . The terms $\nu_{\mathrm{y}}(\mathrm{p} \times 1)$ and $\nu_{\mathrm{x}}(\mathrm{q} \times 1)$ are intercept terms for the indicator variables, and $\alpha(\mathrm{m} \times 1)$ is a vector of effects for the structural equations of $\eta$. All of the other terms and assumptions remain as before, except as follows. First, it is no longer assumed that $\mathrm{E}(\xi)=$ $\mathrm{E}(\eta)=0$. The mean of $\xi, \mathrm{E}(\xi)$, is a parameter known as $\kappa(n \times 1)$ (i.e., effects due to $\xi)$. Second, $\epsilon$ is no longer assumed to be uncorrelated with $\delta$. In general, for a single-sample analysis, the mean parameters $\nu_{\mathrm{y}}, \nu_{\mathrm{x}}$, $\alpha$, and $\kappa$ cannot be identified (i.e., cannot be estimated) without the imposition of numerous constraints (which will not be discussed here). In a multiple-sample analysis, however, simple constraints can be imposed to identify all mean parameters.

If the LISREL computer program is to be used, certain adaptations need to be made in the LISREL model. All indicators ( $y$ and $x$ ) are treated as $y$ variables, all latent variables ( $\eta$ and $\xi$ ) are treated as $\eta$ variables, and a single fixed $x$ variable is set equal to 1 (i.e., a dummy variable). The LISREL model is reformulated as

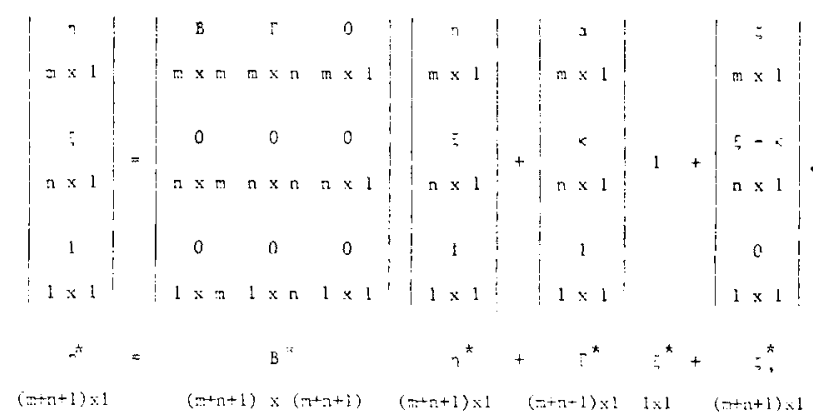




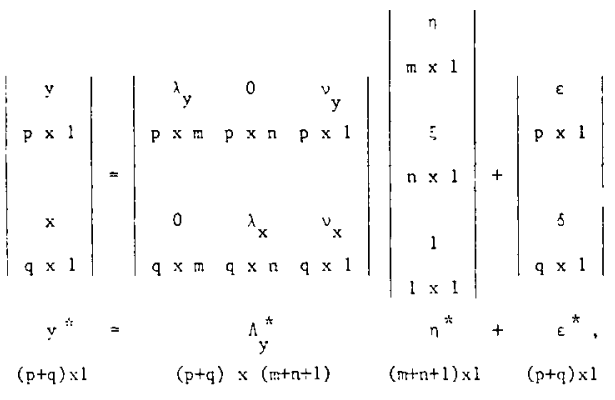

and the remaining parameter matrices are as follows: $\Psi^{*}=\mathrm{D}\left(\sigma_{\zeta}{ }^{2}, \sigma_{\xi}{ }^{2}, 0\right)$ of dimension $(\mathrm{m}+\mathrm{n}+1) \times(\mathrm{m}+\mathrm{n}$ $+1) ; \Phi^{*}=1 ;$ and $\Theta_{\epsilon}{ }^{*}=\left|\Theta_{\epsilon} ; \Theta_{\delta}\right|$ of dimension $(p+q)$ $\mathrm{x}(\mathrm{p}+\mathrm{q})$, where the asterisks indicate reformulated matrices and vectors to be used as input to the program.

Let us examine the conditions and constraints that need to be imposed for the two-sample situation. The matrix to be analyzed must be the moment matrix, rather than the correlation or the covariance matrix. The moment matrix can be computed by the LISREL program through one of the following options: (1) input the raw data, including the fixed $x$ variable set equal to 1 for each case; or (2) input the covariance matrix, including a dummy row of zeros, and a vector of means with a dummy mean of 1 (i.e., for the fixed $x$ variable); or (3) input the correlation matrix with the standard deviations included, which yields a covariance matrix that enables one to follow Option 2. In order to estimate $\alpha$ and $k$, which are estimates of sample differences, the elements of $\alpha$ and $\kappa$ must be fixed to 0 for one of the groups. Otherwise, the model will not be identified. The estimates are interpreted then as effects due to membership in the second group. Although Jöreskog and Sörbom (1983) specified that starting values must be given for all of the free $\Lambda_{y}$ parameters, experience has shown that computer time and estimation problems are minimized when starting values are given for all free parameters. For more than two samples, the model must be reestimated by changing the restrictions on $\alpha$ and $\kappa$, depending on the sample for which the effect estimates are desired.

\section{GUIDE TO MULTIPLE-SAMPLE MODELING}

The multiple-sample-modeling guide represents a suggested sequential strategy for models to be tested in the multiple-sample situation. It is assumed that the reader has set up the problem by following the previously described procedures. Directions are as follows.

Model 1-Construct a theoretically based LISREL model and test it on each sample independently. This provides some indication of possible group differences. At this step, a "final" LISREL model that will be used in the multiple-sample analyses should be selected. Minor modifications in the model may still be made (see Lomax, 1982, for the single-sample guide). The remaining models analyze all samples simultaneously.

Model 2-Beginning with this model and continuing through Model 4, if a model fails to improve on the fit of a previously tested model, in the order indicated, proceed to Model 5 with the best-fitting model thus far in hand. In addition, each model builds upon prior models by keeping previously established equality constraints in the model (e.g., if $\Lambda_{\mathrm{y}}$ is found to be equal across groups, always maintain that condition). Model 2 is a test of equality of the factor-loading estimates in $\Lambda_{\mathrm{y}}(\mathrm{g})$ and in $\Lambda_{\mathrm{x}}(\mathrm{g})$. A test for $\Lambda_{\mathrm{y}}$ and for $\Lambda_{\mathrm{x}}$ equality may be made separately, depending upon the results obtained (i.e., $\Lambda_{\mathrm{y}}$ may be equivalent but not $\Lambda_{\mathrm{x}}$, and vice versa).

Model 3-Test for equality of the uniqueness terms in $\Theta_{\epsilon}(\mathrm{g})$ and in $\Theta_{\delta}(\mathrm{g})$, which may also be examined separately. If Models 2 and 3 yield reasonably good fits, then the measurement models are equivalent across groups.

Model 4-Test for equality of the latent independent variable variance-covariance terms in $\Phi(\mathrm{g})$.

Model 5-Test for equality of the structure coefficients in $\mathrm{B}(\mathrm{g})$ and in $\Gamma^{(\mathrm{g})}$, which may also be examined separately. The user may also want to test for equality of the disturbance terms in $\Psi(\mathrm{g})$.

Model 6-Test for equality of the sample variancecovariance matrices. This is a global (omnibus) test that $\Sigma$ is equal across groups, and is carried out by setting $\Lambda_{\mathrm{x}}(\mathrm{g})=$ I (identity matrix), $\Theta_{\delta}(\mathrm{g})=0$, and testing for equality of $\Phi(\mathrm{g})$, which is a symmetric free matrix. If the $\Sigma$ are found to be equivalent, then all of the LISREL parameters will be equivalent across groups.

Model 7-Here, the best-fitting model is taken from the previous steps and applied to the structured-means situation. This model yields estimates of the intercept terms in $\nu_{\mathrm{y}}, \nu_{\mathrm{x}}, \alpha$, and $\kappa$.

These are the basic set of models that one might investigate, although other variations and submodels may be of interest.

An example of such an analysis process is as follows. Data were taken from the High School and Beyond (National Opinion Research Center, 1980) study, in which a comparison of public $(\mathrm{N}=13,037)$ and private $(\mathrm{N}=2,099)$ school seniors was undertaken. A description of the structural model proposed is shown in Figure 1. The latent variables are home background, academic orientation, extracurricular activity, educational and occupational aspiration, and present achievement. The indicator variables are numerous and are not described here (see Lomax, 1983). The best-fitting single-sample model yields a normed fit index $(\Delta)$ of .885 for the public sample $\left[\chi^{2}(393)=13,290.02\right]$ and .876 for the private sample $\left[\chi^{2}(393)=2,469.51\right]$. The index $\Delta$ is scaled from 0 to 1 and indicates the degree of improvement of fit over the initial null model of subsequent models (see Bentler \& Bonett, 1980, for further discussion of $\Delta$ ). A summary of the multiplesample models tested is shown in Table 1. 


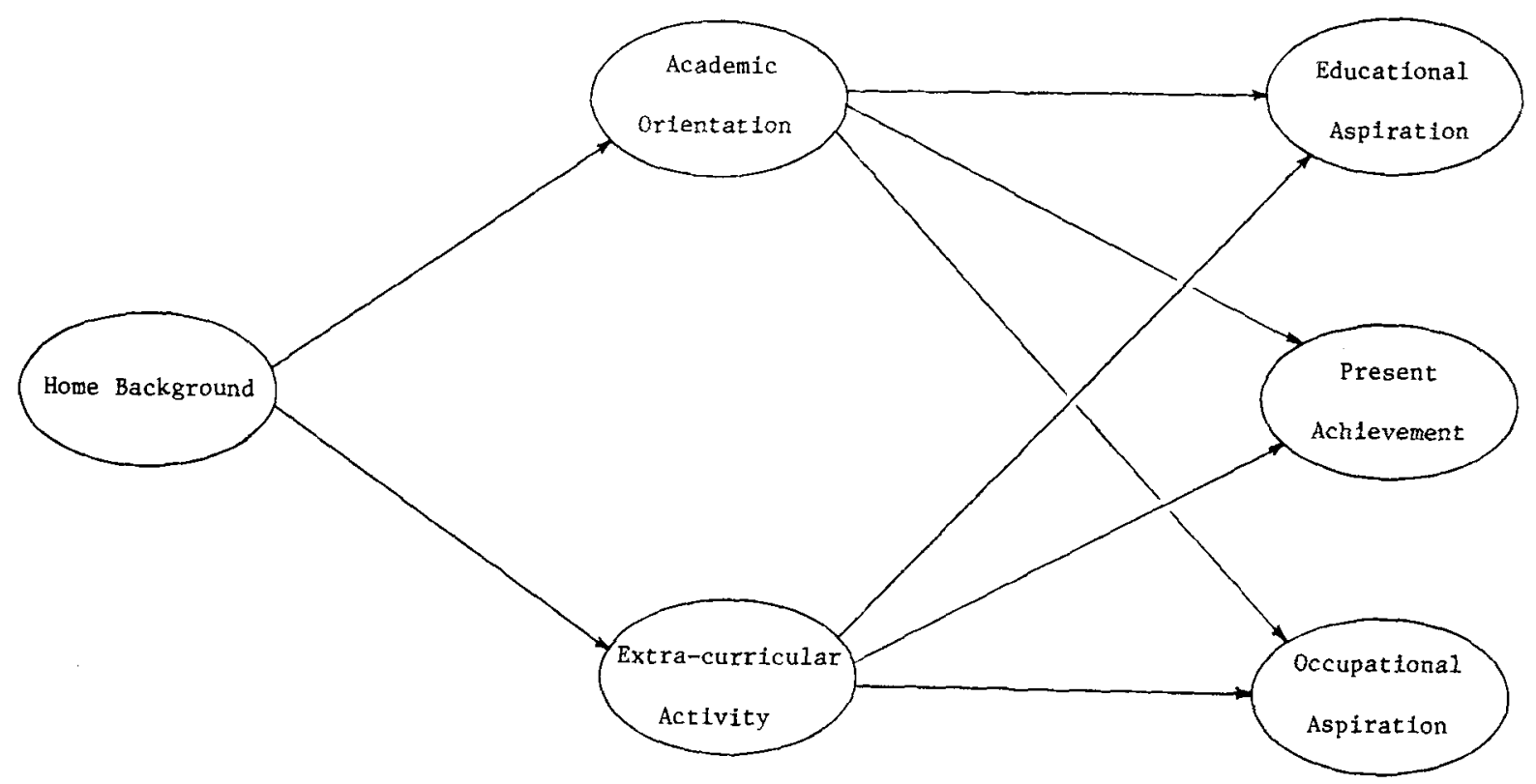

Figure 1

A Theoretical Model of Schooling

Table 1

A Summary of the Multiple-Sample Models

\begin{tabular}{|c|c|c|c|}
\hline Model & $x^{2}$ & Degrees of Freedom & $\Delta$ \\
\hline A-Null & $135,420.52$ & 870 & \\
\hline$B-\Lambda$ equal & $54,914.49$ & 838 & .59 \\
\hline$C-\Lambda, \Theta$ equal & $56,635.37$ & 866 & .58 \\
\hline$D-\Lambda, B, \Gamma$ equal & $30,106.53$ & 830 & .78 \\
\hline $\mathrm{E}-\Lambda, \mathrm{B}, \Gamma$ equal, with correlated $\Theta$ & $16,344.77$ & 818 & .88 \\
\hline F-Structured means & $17,450.45$ & 842 & .87 \\
\hline
\end{tabular}

In the null model (Model $\mathrm{A}$ ), which had no equality constraints, only the measurement error (or unique) variance terms were estimated for each indicator variable. This model serves as a comparison point for the other models. A test of equal-factor-loading matrices (Model B) proved to be a significant improvement in fit over the null model. However, when equality of the uniqueness variances was added to the model (Model $C$ ), the fit failed to improve. At this point, only the $\Lambda$ values may be said to be equal for the two samples. In subsequent models (Models D and E), the structure coefficients were also found to be equal across samples. All of the structure coefficients were found to be significantly different from $0(p<.01)$. Model $E$ allowed for correlated measurement errors, whereas Model D did not, indicating a significantly better-fitting model, as shown by the $\chi^{2}$ and $\Delta$ values.

The structured-means model (Model F) imposed the same equality constraints as Model E, but additionally estimated the intercept terms. Of major importance to the study were the significant effects $(p<.01)$ due to school type. These estimates and their standard errors are shown in Table 2 . The results are as follows:
(1) Those students attending private institutions had a substantially better home background than did those attending public schools; (2) for both academic orientation and extracurricular activity, the values from the private-school sample exceeded those from the publicschool sample when home background was controlled for; and (3) for educational and occupational aspiration and for present achievement, the public-school seniors attained higher levels than did the private-school stu. dents when academic orientation, extracurricular activity, and home background were controlled for. Thus, the model of schooling applied equally well to both the

Table 2

Model F: Effects of School Type

\begin{tabular}{lrc} 
Effects of School Type & Estimate & $\begin{array}{c}\text { Standard } \\
\text { Error }\end{array}$ \\
\hline Home background & 4.857 & .223 \\
Academic orientation & .761 & .040 \\
Extracurricular activity & .328 & .060 \\
Educational aspiration & -.453 & .078 \\
Occupational aspiration & -2.132 & .368 \\
Present achievement & -.533 & .109 \\
\hline
\end{tabular}


public- and the private-school seniors. However, the superiority of the private-school seniors was not a consistent finding, as others previously have reported (e.g., Coleman, Hoffer, \& Kilgore, 1982).

\section{SUMMARY}

Discussed above were: (1) the simple multiple-sample case, in which equality constraints may be imposed on the covariance structure of the measurement and/or structural equation models across samples; (2) the structured-means multiple-sample case, in which constraints may be extended to the mean structure of these models across samples, and thereby allow an assessment of group differences; and (3) a sequential strategy for dealing with the multiple-group situation, illustrated through a model of public and private schools. The author hopes that researchers can use the general LISREL model in the multiple-sample situation to assess group differences, treatment effects, and the like.

\section{REFERENCES}

Bentuer, P. M. (1980). Multivariate analysis with latent variables: Causal modeling. Annual Review of Psychology, 31, 419-456.

Bentler, P. M., \& BonetT, D. G. (1980). Significance tests and goodness of fit in the analysis of covariance structures. Psychological Bulletin, 88, 588-606.

Coleman, J. S., Hoffer, T., \& Kilgore, S. (1982). High school achievement: Public, Catholic and private schools compared. New York: Basic Books.

JöreskoG, K. G. (1978). Structural analysis of covariance and correlation matrices. Psychometrika, 43, 443-477.

JöresKoa, K. G., \& Sönвom, D. (1983). LISREL VI: Analysis of linear structural relationships by maximum likelihood and least squares methods. Chicago: International Educational Services.

Lomax, R. G. (1982). A guide to LISREL-type structural equation modeling. Behavior Research Methods \& Instrumentation, 14, $1-8$.

Lomax, R. G. (1983, April). A structural model of public and private schools. Paper presented at the meeting of the American Educational Research Association, Montreal.

National Opinion Regearch Center. (1980). High school and beyond, information for users, base year (1980) data, version 1 . Chicago: Author.

(Manuscript received October 26, 1983;

revision accepted for publication December $15,1983$. 